\title{
Efficient Steganography Scheme based on Logistic Map and DWT-SVD
}

\author{
Hadis Tarrah \\ Electrical Engineering Dept. \\ Science \& Research branch, \\ Qazvin, Iran
}

\author{
Sattar Mirzakuchaki \\ Electrical Engineering Dept. \\ Iran University of \\ Science \& Technology
}

\begin{abstract}
Steganography is one of the most powerful techniques to conceal the existence of hidden secret message inside a cover medium like image, video, audio, etc. An image is a suitable cover object for steganography because of its great amount of redundant spaces and its abundance. This paper represents a secure steganography scheme which incorporating discrete wavelet transform (DWT) and the stability of the singular values with logistic map-based encryption. In this proposed scheme first of all, the secret image has been encrypted using the logistic map. The encrypted image is added to singular value of original image after two-level DWT to form the stego-image. In this paper the Peak Signal to Noise Ratio (PSNR), correlation coefficient (CR) are used to evaluate the transparency and security of algorithm.
\end{abstract}

\section{Keywords}

Steganography, DWT, SVD, Chaotic map, Logistic Map

\section{INTRODUCTION}

Nowadays, information is converted from paper type to digital format. Therefore, security enhancement in data saving and exchanging is important.

Different methods of cryptography are used for data encryption but all of these techniques can be recognized by invaders. If the information can be embedded in a cover in such a way that it cannot be seen easily, it will not raise the suspicion of invaders. This is the main idea of steganography.

In other words, steganography is the art of embedding information in a cover medium such that it cannot be observable easily. Many different file formats can be used in this regard but digital images are the most popular cover [1, 2]. In steganography, a secret message which is known only to the rightful owner is embedded in the cover medium and is later extracted on the receiving end to authenticate the rightful owner [3].

Steganography methods can be classified into two main categories: spatial domain and transform domain. Spatial domain methods embed massage via direct modification of the pixel values of image and are simpler and less robust against various attacks [4], whereas, in transform domain schemes, the original image first transformed using one of the available transforms like Discrete wavelet transform (DWT), discrete Fourier transform (DFT) and discrete Cosine transform (DCT) etc.

Thereafter the secret message is embedded and inversetransformed to get the stego- image.

The main advantages of Transform Domain are more information embedding and more robustness against various attacks. But the computational cost is higher than spatialdomain methods $[5,6]$. In DWT method, message is embedded by modulating the magnitude of coefficients in a transform domain and is compatible by Human Visual System.

Steganography using DWT has more advantages over DCT because of high compression ratios and also avoidance of interferences due to artifacts. So DWT is a better technique for hiding confidential data [7]. On the other hand, Better approach in terms of transparency and invisibility is a combination of DWT-SVD steganography technique.

The Discrete Wavelet Transform can identify portions of cover image where message can be imperceptibly embedded. Also, one of the main properties of SVD is that slight variations of singular values do not affect the visual properties of the cover image, which results good transparency and robustness.

In this paper Combination of discrete wavelet transform (DWT) and singular value decomposition (SVD) have been used to achieve invisibility and transparency.

In proposed steganography scheme secret images are embedded by modifying the singular values in LH and HL bands of the cover image after two level decomposition.

The encryption method which is used for the secret image is logistic map which is a kind of chaotic map [8].

The rest of the paper is organized as follows: In section 2, 3 and 4 are discussed chaotic map, DWT and SVD respectively. Proposed algorithm is introduced in section 5. In section 6 simulation result is shown and values of peak signal to noise ration and correlation coefficient are calculated. Finally section 7 is described conclusion.

\section{CHOTIC MAP}

Chaos theory is a branch of mathematics and Chaotic Systems are basically nonlinear dynamical systems which exhibiting random behavior for certain range of values of system parameters.

Many properties of chaotic systems such as no periodicity, easy generation, and sensitivity to the initial conditions can be applied to security processes. Chaos-based image encryption methods are very useful for protecting the contents of digital images [9].

Logistic map is one of the discrete chaotic systems which can exhibit strange attractors. Logistic map is widely used for designing digital chaotic cryptosystems $[8,11]$.

In logistic map iterates are generated using the below equation:

$X_{n+1}=r X_{n}\left(1-X_{n}\right)$

In which the $X_{n} \in[0,1]$. According to research requirements for image Encryption, the logistic map chaotic with the primary value of $X_{0}=0.3$ and $\mathrm{r} \in[3.57,4]$ are used. 


\section{DISCRETE WAVELET TRANSFORM}

The DWT split an image into four sub bands: lower resolution component (LL) after the low-pass filtering both the rows and columns which contains a rough description and without details of image, horizontal detail (HL) and vertical detail component (LH) which is the results of low-pass filtering on one direction and high-pass filtering in the other direction and diagonal detail components $(\mathrm{HH})$ after high-pass filtering in both directions and contains details about the edge components [12].

DWT decomposition plays an important role in the image processing field because of modifying the areas of image which is corresponding with modified DWT coefficients of image.

LL sub band contain general details and most of the energy of image is concentrated in it, so embedding in LL can degrade the image however, improves the robustness. Whereas in high frequency sub band $\mathrm{HH}$ include edges of the image and human eyes is less sensitive to changes in such sub band. Therefore in order to enhance robustness and invisibility, message embedding should be done in the intermediate frequency bands i.e. HL and LH after two level DWT decomposition.

Figure 1 shows the DWT decomposition.

\begin{tabular}{|c|c|c|}
\hline \multirow{2}{*}{$\mathrm{LL}^{1}$} & $\mathrm{LL}^{2}$ & $\mathrm{HL}^{2}$ \\
\cline { 2 - 3 } & $\mathrm{LH}^{2}$ & $\mathrm{HH}^{2}$ \\
\hline $\mathrm{LH}^{1}$ & \multicolumn{2}{|c|}{$\mathrm{HH}^{1}$} \\
\hline
\end{tabular}

Fig 1: DWT decomposition

\section{SINGULAR VALUE \\ DECOMPOSITION}

An image can be viewed as a matrix with nonnegative scalar entries. If $\mathrm{A}$ be a matrix with size $\mathrm{m} \times \mathrm{n}$ its $\mathrm{SVD}$ is given by $\mathrm{I}$ $=U S V^{T}$, where $\mathrm{U}$ and $\mathrm{V}$ are orthogonal matrices, and $\mathrm{S}=$ $\operatorname{diag}(\alpha \mathrm{i})$ is a diagonal matrix of singular values $\alpha \mathrm{i}, \mathrm{i}=1 \ldots \mathrm{n}$. The columns of $\mathrm{U}$ and $\mathrm{V}$ are the left and right of singular vectors of A respectively $[9,12]$.

There are advantages of using SVD in digital image processing. SVD transformation can be applied to an image with optional sizes such as square or rectangular also singular values are less affected in image processing [13].

\section{PROPOSED ALGORITHM}

In the proposed algorithm, first of all, one must perform onelevel DWT on secret image and then use of logistic map to encrypt it as follows:

(1) Perform one-level DWT on Secret image

\begin{tabular}{|c|c|}
\hline $\mathrm{ll}$ & $\mathrm{hl}$ \\
\hline $\mathrm{lh}$ & $\mathrm{hh}$ \\
\hline
\end{tabular}

Fig 2: secret image

(2) Use logistic map to generate $n * m$ (size 11) random number

(3) Sort generated numbers as descend
(4) Reshape 11 matrix in one raw and use of above index to change pixels order

(5) Apply IDWT on image to obtain encrypted image

(6) Divide encrypted image $(\mathrm{m} \times \mathrm{n})$ into 4 parts:

$C=\left[\begin{array}{ll}C_{1} & C_{2} \\ C_{3} & C_{4}\end{array}\right]$

$C_{1}=C\left(1: \frac{M}{2}, 1: \frac{N}{2}\right)$

$C_{2}=C\left(1: \frac{M}{2}, \frac{N}{2}+1: N\right)$

$C_{3}=C\left(\frac{M}{2}+1: M, 1: \frac{N}{2}\right)$

$C_{4}=C\left(\frac{M}{2}+1: M, \frac{N}{2}+1: N\right)$

\subsection{Embedding}

Image is embedded in the LH and HL sub bands of the cover image after two level DWT decomposition. The embedding is done by modifying the singular values in $\mathrm{LH}$ and $\mathrm{HL}$ sub bands.

(1) Perform two-level Discrete Wavelet Transform on cover image.

\begin{tabular}{|c|c|c|c|}
\hline \multicolumn{2}{|c|}{ LL } & LL1 & HL1 \\
\cline { 4 - 4 } \multicolumn{2}{|c|}{} & LH1 & HH1 \\
\hline LL2 & HL2 & \multicolumn{2}{|c}{ HH } \\
\hline LH2 & HH2 & \multicolumn{2}{|c}{} \\
\hline
\end{tabular}

Fig 3: Two-level DWT

(2) Apply Singular Value Decomposition to LH1 and HL1 \& LH2 and HL2 sub bands, i.e.,

$A_{i}=U_{i} S_{i} V_{i}^{T} \quad i=1,2,3,4$

(3) Modify the singular values in LH1, HL1, LH2, HL2 sub bands with quarter of the encrypted image from equation (2) and scale factor $\gamma$, i.e.,

$$
U S W_{W_{i}}=S_{i}+\gamma * C_{i} \quad i=1,2,3,4
$$

(4) Apply SVD to them, respectively, i.e.

$\left[U_{W_{i}}, S_{W_{i}}, V_{W_{i}}\right]=\operatorname{SVD}\left(\mathrm{USV}_{\mathrm{Wi}_{\mathrm{i}}}\right) \mathrm{n}=1,2,3,4$

(5) Using $\mathrm{U}_{\mathrm{i}}, \mathrm{V}_{\mathrm{i}}$ from step 2 and $\mathrm{S}_{\mathrm{W}_{\mathrm{i}}}$ from step 4, obtain:

$A_{i}^{*}=U_{i} S_{W_{i}} V_{i}^{T} \quad i=1,2,3,4$

(6) By using LL1, HH1, A,$A_{2}^{*}$ and applying IDWT, will have LH_new and by using LL2, HH2, $A_{3}^{*}, A_{4}^{*}$ and applying IDWT, HL_new will be achieved.

(7) Applying IDWT on HH, LL, LH_new and HL_new to obtain $\mathrm{A}_{\mathrm{w}}^{*}$ which is stego-image.

(8) Sending $A_{W}^{*}, U_{W_{i}}, V_{W_{i}} i=1,2 \ldots 8$ to receiver.

\subsection{Extracting}

(1) Applying one-level DWT on $A_{w}^{*}$ to obtain LLnew, LHnew, HLnew, HHnew.

(2) Again apply one-level DWT on middle frequency ( $\mathrm{LH}$, HL) to obtain 8 sub bands which are: 


\section{LLnew1, LHnew1, HLnew1, HHnew1,} LLnew2, LHnew2, HLnew2, HHnew2

(3) Applying SVD on intermediate frequency of last step, according to below equation:

$A^{*}{ }_{i}=U^{*}{ }_{i} S_{W_{i}}^{*} V^{* T}{ }_{i} \quad i=1,2,3,4$

Which $\mathrm{i}=1,2,3,4$ is related to LHnew1, HLnew 1 , LHnew2, HLnew2 accordingly.

(4) By using $S_{W_{i}}^{*}$ and $U_{W_{i}}, V_{W_{i}}$ which is sent and according to below equation, will be resulted:

$D_{i}^{*}=U_{W_{i}} S_{W_{i}}^{*} V_{W_{i}}^{T} \quad i=1,2,3,4$

(5) Extract quarters of secret image:

$S Q_{i}^{*}=\frac{\left(D_{i}^{*}-S_{w i}^{*}\right)}{\gamma} i=1,2,3,4$

(6) Combine quarters to obtain the cipher image i.e.

$$
S Q=\left[\begin{array}{cc}
S Q_{1}^{*} & S Q_{2}^{*} \\
S Q_{3}^{*} & S Q_{4}^{*}
\end{array}\right]
$$

Finally in the decryption of Cipher-image, below items should be done for achieving to secret message.

(1) Apply DWT on SQ of equation (10) to obtain $4 \mathrm{sub}$ bands

(2) sort each sub band with logistic map

(3) Perform IDWT on sub bands to obtain secret image.

\section{SIMULATION RESULTS}

The proposed is demonstrated using MATLAB. In order to test the proposed algorithm, a gray level image "Lena" of size $512 * 512$ is used as cover image and cameraman image of size $256 * 256$ is selected as secret image.

The results are shown in Fig.4.

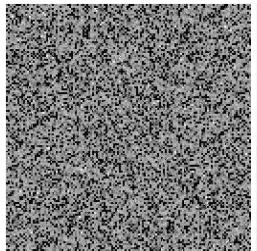

(a)

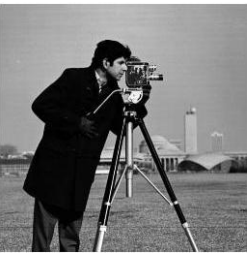

(b)

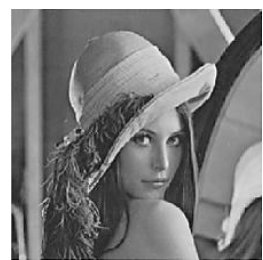

(d)

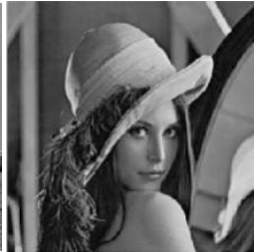

(c)

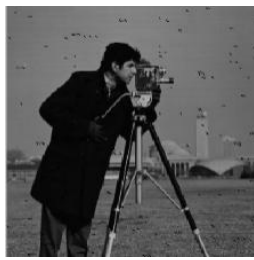

(e)
Fig.4 (a) cover image, (b) secret image, (c) encrypted secret image, (d) embedded image, (e) decrypted extracted image

As a result of embedding, Peak Signal to Noise Ratio (PSNR) is chosen for detecting transparency. Figure 4(d) with $\gamma=0.05$ and PSNR $=45$ keeps a good similarity because where PSNR is higher than $30 \mathrm{~dB}$, it is hard to distinguished between original image and reconstructed one so this algorithm keeps good transparency. By using logistic map in figure 4(c), correlation coefficient between pairs of encrypted adjacent pixels is -0.12 , so this algorithm also provides good security. Also the Normalized Correlation (NC) for secret image before and after steganography is 0.59 (figure $4(\mathrm{e})$ ) which gives a measure of the visibility of steganography and its peak value is 1 .

To investigate the robustness of the algorithm, the stegoimage is subjected to various types of attacks such as salt \& pepper noise, Cropping, compression, scaling \& rotating. As a quantitative measure of the degradation effect caused by the attacks, PSNR is used. PSNR values indicate lower degradation, hence indicating that this steganography technique is robust to various attacks.

Table 1 shows PSNR of stego-image against various attacks.

Table 1. Attacks applied on stego-image

\begin{tabular}{|c|c|}
\hline Attacks & PSNR \\
\hline Salt \& Pepper noise & 40.56 \\
\hline Scaling 0.75 & 30.99 \\
\hline Compression70\% & 39 \\
\hline Rotating 30 & 27.25 \\
\hline Cropping 50\% & 27.01 \\
\hline
\end{tabular}

\section{CONCLUSION}

This paper deals with a DWT-SVD steganography scheme based on logistic map in which secret image is embedded in the HL and LH sub bands of the cover image after two level DWT decomposition by modifying the singular values of the cover image with encrypted secret image.

The performance was evaluated using PSNR, correlation coefficient and Normalized Correlation. As simulation results show this method provides good transparency and security. Also as Table 1 shows has good robustness against various types of attacks.

In future should be focused on $\mathrm{NC}$ of secret image to have no visible difference with original image after extracting. Also can use of the other type of images like fractal images and apply this algorithm on them.

\section{REFERENCES}

[1] Morkel, T., Eloff, J., and Olivier, MS., "An Overview of Image Steganography", Proceedings of the Fifth Annual Information Security South Africa Conference (ISSA), Sandton, South Africa. June/July 2005

[2] Mahmoudpour, S., and Mirzakuchaki, S., "Hardware Architecture for a Message Hiding Algorithm with Novel Randomizers", International Journal of Computer Applications, Volume 37, No.7, 2012.

[3] Singh, P., Shivani, S. and Agarwal, S., "A Chaotic Map Based DCT-SVD Watermarking Scheme for Rightful Ownership Verification", IEEE, August 2014

[4] Nikolaidis, N. and Pitas, I., "Robust image watermarking in the spatial domain", Signal Processing, vol. 66, pp. 385-403, 1998

[5] Prodilchuk, C., De1p, E. "Digital watermarking algorithms and applications", IEEE Signal Processing Magazine, 2001, 69 (13):33-46 
[6] Desheng, X., Yueshan, X., "Image watermarking algorithm based on DWT". Computer Engineering and Design, 2005, 26(3)611-613.

[7] Babya, D., Thomasa, J., Augustinea, G., Georgea, E., Michaela, N., "A Novel DWT based Image Securing Method using Steganography", International Conference on Information and Communication Technologies (ICICT), 2014.

[8] Kushwah, N., Sharma, M., "Chaotic Map based Block Encryption", International Journal of Computer Applications, Volume 71- No.16, 2013

[9] Jahan, R., "Efficient and Secure Digital Image Watermarking Scheme using DWT-SVD and Optimized Genetic Algorithm based Chaotic Encryption", International Journal of Science, Engineering and Technology Research (IJSETR) Volume 2, Issue 10, October 2013
[10] Patidar, V., and Sud, K., "A Pseudo Random Bit Generator Based on Chaotic Logistic Map and its Statistical Testing”, Informatica 33, (2009), 441-452 441

[11] Lei, B., Ni, B., Chen, S., Wang, T., Zhou, F., "Optimal image watermarking scheme based on chaotic map and quaternion wavelet transform", Springer, Nonlinear Dyn (2014)

[12] Joseph, A., Anusudha, K., "Robust watermarking based on DWT SVD", International Journal of Signal \& Image Processing, Issue. 1, Vol. 1, October 2013

[13] Lai, C., Tsai, C., "Digital image watermarking using discrete wavelet transform and singular value decomposition," IEEE Transactions on Instrumentation and Measurement, vol. 59, No.11, pp. 3060-3063, Nov. 2010 . 